\title{
Silencing long non-coding RNA, differentiation antagonizing non-protein coding RNA promotes apoptosis and inhibits tumor growth in colon cancer
}

\author{
XIAO-JIN YANG ${ }^{1}$, JING-JING ZHAO ${ }^{1}$, WEI-JUN CHEN ${ }^{1}$, GEN-GEN ZHANG $^{2}$, WEI WANG ${ }^{3}$ and HAN-CHUAN TAO ${ }^{1}$ \\ Departments of ${ }^{1}$ General Surgery, ${ }^{2}$ Pathology, and ${ }^{3}$ Clinical Laboratory, Dongtai Municipal People's Hospital, \\ Nantong University, Dongtai, Jiangsu 224200, P.R. China
}

Received December 11, 2017; Accepted April 19, 2018

DOI: $10.3892 / \mathrm{ol} .2018 .9034$

\begin{abstract}
The long non-coding RNA (lncRNA) differentiation antagonizing non-protein coding RNA (DANCR) has been reported to be a novel potential biomarker for colon cancer prognosis. However, its functional role in colon cancer remains unknown. In the present study,DANCR expression in colon cancer cell lines was determined by reverse transcription-quantitative polymerase chain reaction. Cell Counting kit- 8 assay, colony formation assay, flow cytometry, Hoechst 33258 staining and western blotting were utilized to investigate the effect and mechanism of DANCR in the regulation of colon cancer growth. Establishment of a xenograft tumor model followed by terminal deoxynucleotidyl transferase (TdT) dUTP nick-end labeling assay and immunohistochemical staining were performed to confirm the findings in vitro. DANCR was revealed to be overexpressed in all human colon cancer cell lines. Silencing DANCR by small interfering RNA significantly inhibited cell proliferation and colony formation. Flow cytometry analyses and Hoechst 33258 staining revealed that apoptosis was induced upon DANCR-knockdown. Silencing DANCR was revealed to efficiently impair colon tumor growth by promoting caspase 3 expression and tumor apoptosis. In summary, the results of the present study demonstrated that DANCR is a potential therapeutic target in colon cancer.
\end{abstract}

\section{Introduction}

Colon cancer is the third most common type of cancer and the fourth leading cause of cancer-associated mortality worldwide $(1,2)$. Multiple factors are involved in the occurrence and development of colon cancer, including uncontrolled cell proliferation, angiogenesis and suppression of apoptosis (3-5).

Correspondence to: Dr Han-Chuan Tao, Department of General Surgery, Dongtai Municipal People's Hospital, Nantong University, 2 Kangfu West Road, Dongtai, Jiangsu 224200, P.R. China

E-mail: taohanchuan@126.com

Key words: colon cancer, long non-coding RNA, differentiation antagonizing non-protein coding RNA, apoptosis
Mutations are present in almost all types of colon cancer, and point mutations in the KRAS gene can lead to activation of potentially carcinogenic RAS protein activity (6). Therefore, understanding the pathogenesis of colon cancer and elucidating the functional role of point mutations in different genes are imperative to improving treatment strategies for patients.

Non-coding RNAs (ncRNAs) have no protein-coding abilities but may act as key regulators to control biological and pathological processes (7). A large number of ncRNAs that function as central orchestrators of cell-specific gene networks have been identified (8). An important subclass of these ncRNAs are long non-coding RNAs (lncRNAs) that are broadly defined as regulatory non-coding transcripts of longer than 200 nucleotides. Thus far, $>100,000$ lncRNA genes have been described in the human genome, which is greater than the number of protein-coding and microRNA genes combined. Although their biological roles and mechanisms of function remain largely elusive, lncRNAs have critical roles in the development process, cellular homeostasis, genomic imprinting and pluripotency of embryonic stem cells (9). The importance of IncRNA regulation has been emphasized by their roles in the etiology of human diseases (10-13). Several lncRNAs are involved in carcinogenesis, disease progression, metastasis and/or chemoresistance of human cancer types $(14,15)$. Thus, lncRNAs are novel therapeutic targets in cancer.

The novel lncRNA differentiation antagonizing non-protein coding RNA (DANCR) is located on human chromosome 4, with the closest adjacent annotated genes located $54.8 \mathrm{~kb}$ upstream of USP46 and $28.7 \mathrm{~kb}$ downstream from ERVMER34-1 and the ANCR locus (14). Previous studies have demonstrated that DANCR is involved in the differentiation of progenitors and osteoblasts $(16,17)$. Recently, abundant evidence has suggested that DANCR is an oncogene in various types of cancer, including breast cancer (15), gastric cancer (16), prostate cancer (17) and hepatocellular carcinoma (18). In colon cancer, DANCR expression was increased, and high DANCR expression was revealed to be correlated with a poor overall survival (OS) and disease-free survival (DFS) in patients (19). However, the biological functions and significance of DANCR in colon cancer have not yet been established. In the present study, DANCR expression in colon cancer cell lines was determined by reverse transcription-quantitative polymerase chain 
reaction (RT-qPCR) and Cell Counting kit-8 assay. A colony formation assay, flow cytometry, Hoechst 33258 staining and western blotting were employed to investigate the effects and mechanisms of DANCR in regulating colon cancer growth. Establishment of a xenograft tumor model was followed by terminal deoxynucleotidyl transferase (TdT) dUTP nick-end labeling (TUNEL) assay. Immunohistochemical staining was performed to confirm the findings in vitro.

\section{Materials and methods}

Cancer cell lines and cell culture. Human colon cancer SW620, SW480, HCT116, HT29, HCT15 and Caco-2 cell lines and normal human colon epithelial HCoEpiC cells were obtained from American Type Culture Collection (Manassas, VA, USA). Cells were cultured in Dulbecco's modified Eagle's medium (DMEM) supplemented with 10\% fetal bovine serum (FBS; Invitrogen; Thermo Fisher Scientific, Inc., Waltham, MA, USA), $100 \mathrm{U} / \mathrm{ml}$ penicillin $\mathrm{G}$ and $100 \mu \mathrm{g} / \mathrm{ml}$ streptomycin (Thermo Fisher Scientific, Inc.) in a humidified atmosphere of $5 \% \mathrm{CO}_{2}$ at $37^{\circ} \mathrm{C}$.

Generation of stably infected cell lines. Lentiviral vector-based short hairpin RNA (shRNA) targeting non-specific control (NC) or human LncRNA DANCR (5'-GGAGCTAGAGCA GTGACAATG-3') were purchased from Shanghai Co., Ltd., GenePharma (Shanghai, China). Cells achieved 70-80\% confluence in Lv-shNC and Lv-shDANCR infection. The stably infected cells were selected by adding puromycin $(2 \mu \mathrm{g} / \mathrm{ml})$, and were collected for further qPCR analysis to confirm the efficient knockdown of DANCR in colon cancer cell lines.

RNA isolation and RT-qPCR. Total cellular RNA was isolated from colon cancer cells using TRIzol (Thermo Fisher Scientific, Inc.) according to the manufacturer's protocols. The RNA was reverse-transcribed into cDNA by using a reverse transcription kit (RR047A; Takara Bio, Inc., Otsu, Japan) according to the manufacturer's protocol. Real-time reverse transcription-PCR was performed with a SYBR Green PCR kit (RR902A; Takara Bio, Inc.) and ABI 7300 Real-Time PCR System (Applied Biosystems; Thermo Fisher Scientific, Inc.). Amplification was performed as follows: Incubation at $95^{\circ} \mathrm{C}$ for $30 \mathrm{sec}$, followed by 40 cycles of denaturation at $95^{\circ} \mathrm{C}$ for $5 \mathrm{sec}$ and subsequent annealing and extension at $58^{\circ} \mathrm{C}$ for $30 \mathrm{sec}$. Relative gene expression was calculated using the $2^{-\Delta \Delta C q}$ method (20). For fold change analysis, GAPDH (forward, 5'-TGCGTGACA TTAAGGAGAA-3' and reverse, 5'AAGGAAGGCTGGAAG AGT-3') expression was analyzed to normalize target gene expression. Primer sequence of DANCR used in this study are as follow (forward, 5'-GCCACTATGTAGAGGGTTTC-3' and reverse, 5'-ACCTGCGCTAAGAACTGAGG-3').

Cell Counting kit-8 assay. Cells (1,000 cells/well) were plated in 96-well plates. Cell proliferation was measured at $0,24,48$ and $72 \mathrm{~h}$ post cell plating. Cell Counting kit- 8 assay (Beyotime Institute of Biotechnology, Beijing, China) was used for cell viability determination at a wavelength of OD $450 \mathrm{~nm}$.

Western blotting. Cells were washed once with ice-cold PBS and lysed in radioimmunoprecipitation assay buffer (Beyotime
Institute of Biotechnology) containing $1 \%$ protease inhibitors (Sigma-Aldrich; Merck KGaA, Darmstadt, Germany). Protein concentration was subsequently measured with a BCA kit (Thermo Fisher Scientific, Inc.), $\sim 10 \mu \mathrm{g}$ protein was separated with 8-12\% SDS-PAGE gel and blotted onto polyvinylidene fluoride or polyvinylidene difluoride membranes (Merck KGaA, Darmstadt, Germany). Membranes were subsequently blocked with $5 \%$ milk at room temperature for $1 \mathrm{~h}$ and incubated with primary antibodies against caspase 3 (1:600; cat. no. 9665), caspase 8 (1:1,000; cat. no. 4790), caspase 9 (1:800; cat. no. 9508), p53 (1:1,200; cat. no. 2524), Cyto C (1:1,000; cat. no. 4280), Bcl-2 (1:1,200; cat. no. 3498), Bcl-xL (1:1,000; cat. no. 2762) and GAPDH (1:5,000; cat. no. 2118) (Cell Signaling Technology, Inc., Danvers, MA, USA) at $4^{\circ} \mathrm{C}$ overnight, followed by 3 washes with tris-buffered saline with tween and a $1 \mathrm{~h}$ incubation with horseradish peroxidase-conjugated secondary antibodies at room temperature (OriGene Technologies Inc., Beijing, China). Protein bands were visualized with an enhanced chemiluminescence kit (Merck KGaA). The density of bands was quantified by ImageJ software (version 1.48; National Institutes of Health, Bethesda, MD, USA).

Apoptosis analysis by flow cytometry. Cells were harvested and stained with Annexin V-FITC/PI Apoptosis Detection kit (Nanjing KeyGen Biotech Co., Ltd., Nanjing, China), according to the manufacturer's protocol. Apoptotic cells were assessed by flow cytometry (De Novo Software, Glendale, CA, USA). Each experiment was performed three times.

Hoechst 33258 staining. A total of $1 \times 10^{5}$ cells were plated in each well of the 6-well plates. After $24 \mathrm{~h}$, the cells were fixed with $4 \%$ paraformaldehyde for $10 \mathrm{~min}$ at room temperature. Following washing with PBS 3 times, the cells were stained with Hoechst 33258 (Beyotime Institute of Biotechnology) in the dark for $15 \mathrm{~min}$ at room temperature. Images of the cells were captured using a fluorescence microscope (Olympus Corporation, Tokyo, Japan).

Colony formation assay. A total of 1,000 cells were plated in each well of the 6-well plates containing $2 \mathrm{ml}$ DMEM medium (10\% FBS). Following 7-10 days, the cells were fixed with $4 \%$ paraformaldehyde for $10 \mathrm{~min}$ at room temperature. Cells were washed with PBS 3 times, and subsequently stained with purple crystal (Beyotime Institute of Biotechnology) for $15 \mathrm{~min}$ at room temperature. The number of colonies were counted and analyzed. The assays were replicated three times.

Animal study. This study was approved by the Ethics Committee of Dongtai Municipal People's Hospital of Nantong University and complied with the animal guidelines. Ten 6-week-old female BALB/c nude mice (weighing between 18 and $20 \mathrm{~g}$ ) were purchased from Hfkbio (Beijing, China; http://www.hfkbio.com/) and housed in a specific pathogen-free room (at a temperature of $22-25^{\circ} \mathrm{C}$ ) with $12 \mathrm{~h}$ dark/light cycle and ad libitum access to food and water. SW480-shNC and SW480-shDANCR cells $\left(5 \times 10^{6}\right.$ cells/mouse $)$ were subcutaneously injected into the right flank of ( $n=5$ per experimental group). Tumor length and width were measured every five days from the tenth day post cell injection. Tumor 


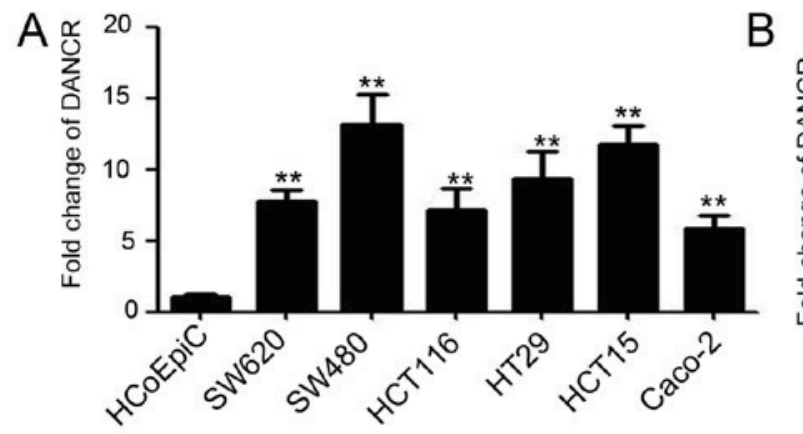

B
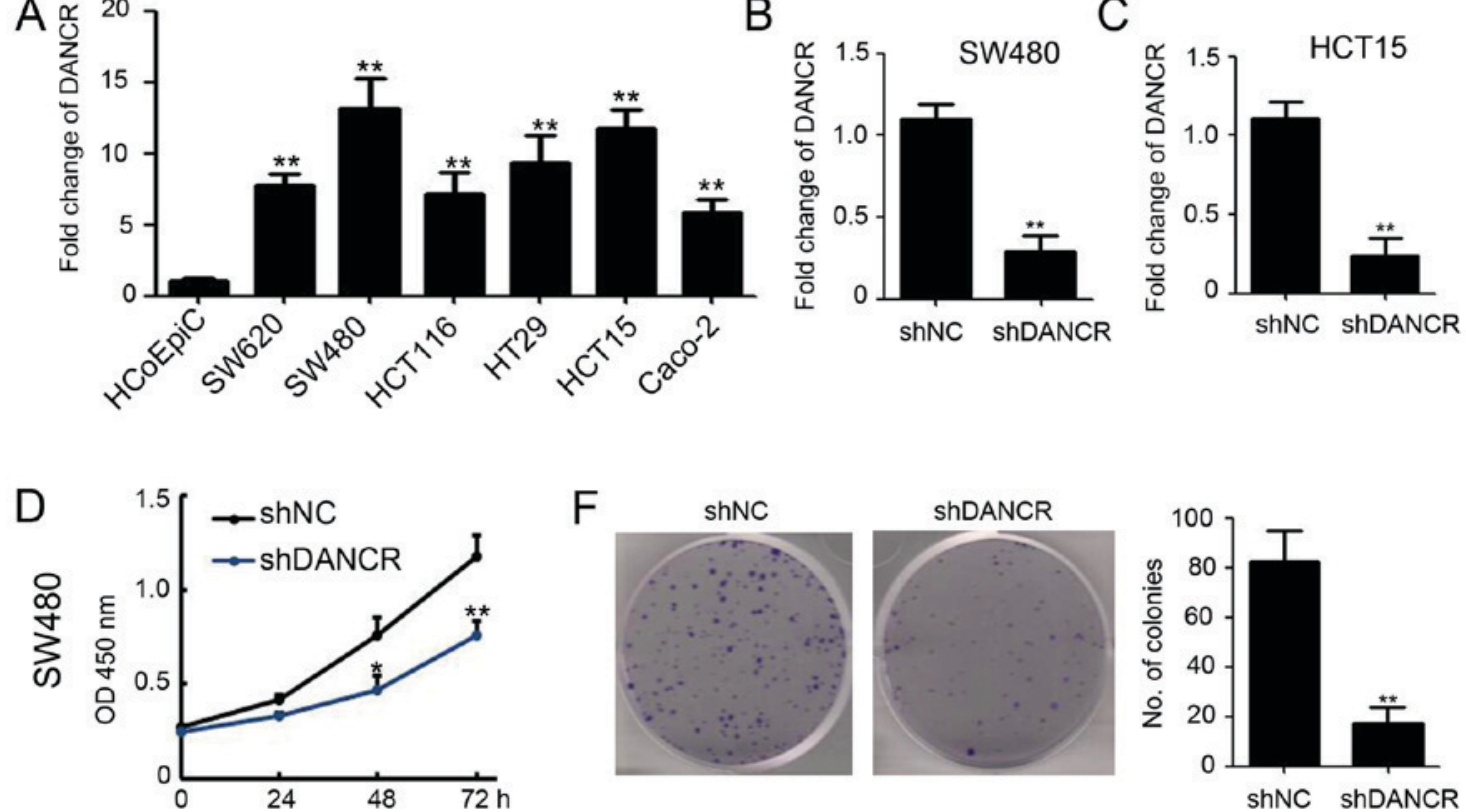

$\mathrm{F}$
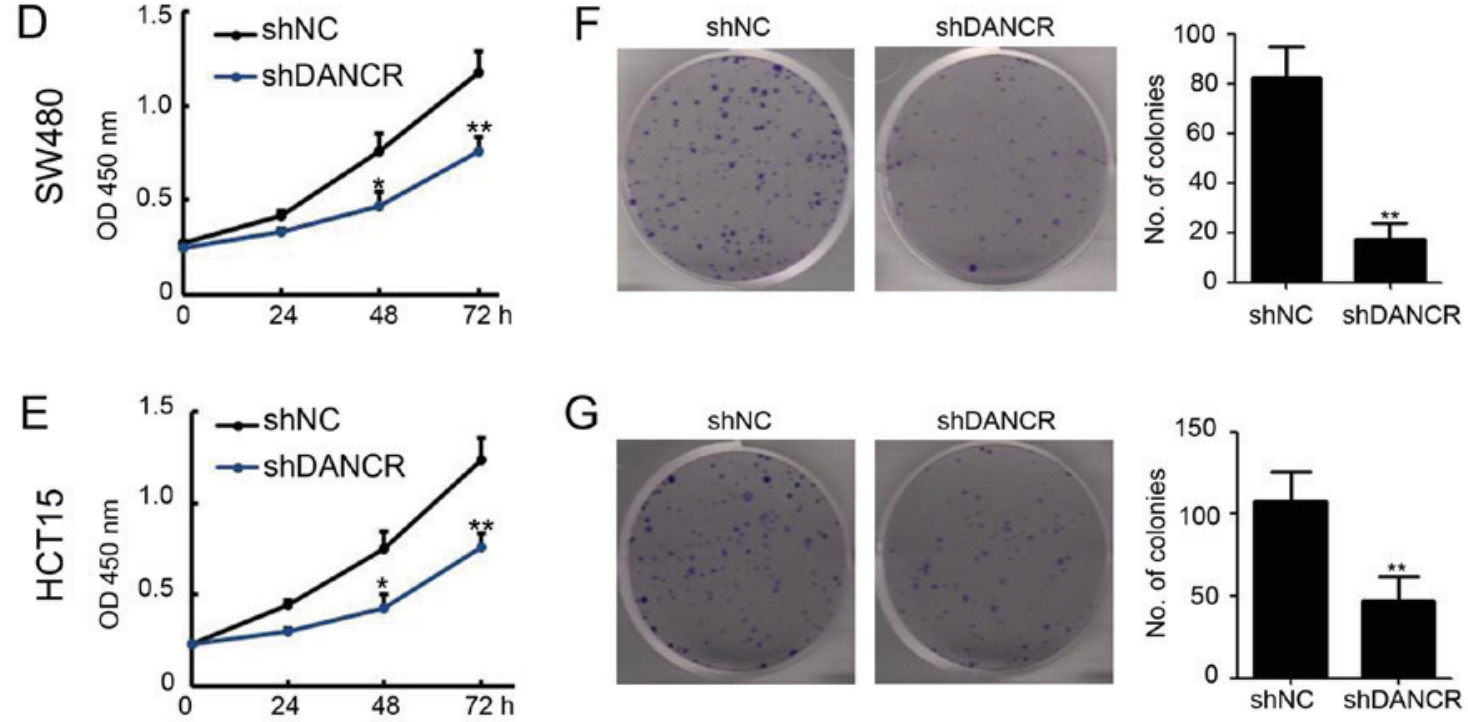

Figure 1. Silencing DANCR inhibits colon cancer cell growth in vitro. (A) Fold change of DANCR in human colon cancer cell lines and normal human epithelial cells (HCoEpiC). DANCR was examined by RT-qPCR and normalized to GAPDH expression ( $\mathrm{P}<0.01)$. (B) SW480 and (C) HCT15 cell lines were infected with lentivirus-based shRNA targeting DANCR and NC. The fold change of DANCR were examined by RT-qPCR and normalized to GAPDH expression $(\mathrm{P}<0.01)$. (D) Cell Counting kit- 8 assay was performed to detect the cell viability of SW480-shNC and SW480-shDANCR cells at $0,24,48$ and $72 \mathrm{~h}$ post cell plating $(\mathrm{P}<0.05, \mathrm{P}<0.01)$. (E) Cell Counting kit-8 assay was performed to detect the cell viability of HCT15-shNC and HCT15-shDANCR cells at $0,24,48$ and $72 \mathrm{~h}$ post cell plating $(\mathrm{P}<0.05, \mathrm{P}<0.01)$. (F) Colony formation assay was performed in $\mathrm{SW} 480$-shNC and $\mathrm{SW} 480$-shDANCR cells. The number of colonies was analyzed $(\mathrm{P}<0.01)$. (G) Colony formation assay was performed in HCT15-shNC and HCT15-shDANCR cells. The number of colonies was analyzed $(\mathrm{P}<0.01)$. ${ }^{*} \mathrm{P}<0.05$ and ${ }^{* *} \mathrm{P}<0.01$ vs. shNC. shDANCR, short hairpin differentiation antagonizing non-protein coding RNA; shRNA, short hairpin ribonucleic acid; $\mathrm{NC}$, negative control; RT-qPCR, reverse transcription-quantitative polymerase chain reaction.

volumes were calculated as ellipsoids (length $\mathrm{x}$ width ${ }^{2} \mathrm{x} 0.52$ ). At 30 days post cell injection, the mice were sacrificed and the tumors were removed and measured.

Terminal deoxynucleotidyl transferase dUTP nick end labeling (TUNEL) assay. Tumor tissue sections were examined for the presence of apoptotic cells using the DeadEnd ${ }^{\mathrm{TM}}$ Fluorometric TUNEL system (Promega Corporation, Madison, WI, USA) according to the manufacturer's protocol, in which fragmented DNA from apoptotic cells is end-labeled with the fluorophore. Following deparaffinization and rehydration, sections were fixed with $4 \%$ paraformaldehyde at room temperature $\left(22-25^{\circ} \mathrm{C}\right)$ for $10 \mathrm{~min}$, permeabilized with proteinase $\mathrm{K}$ for $8-10 \mathrm{~min}$ at room temperature $\left(22-25^{\circ} \mathrm{C}\right)$, and repeatedly fixed. The sections were then covered with $50 \mathrm{ml}$ terminal deoxynucleotidyl transferase mix for $1 \mathrm{~h}$ at $37^{\circ} \mathrm{C}$ in a humidified chamber. The coverslips were removed and the sections were immersed in $2 \mathrm{x}$ saline-sodium citrate buffer for $15 \mathrm{~min}$, washed with PBS and mounted with glycerin (Beyotime Institute of Biotechnology) that included DAPI
(Beyotime Institute of Biotechnology). Four random fluorescence images were captured using a fluorescence microscope (Olympus Corporation).

Immunohistochemical staining. Collected tumor tissues from mice injected with SW480 cells were fixed with $4 \%$ paraformaldehyde at room temperature for $48 \mathrm{~h}$. Then, fixed tumor tissues were embedded in paraffin and sectioned into $4 \mu \mathrm{m}$ slices. Following removal of the paraffin and antigen retrieval in citrate buffer ( $\mathrm{pH} \mathrm{6.0)}$ at high temperature and pressure for $3 \mathrm{~min}$, slices were blocked with $5 \%$ goat serum at room temperature $\left(22-25^{\circ} \mathrm{C}\right)$ for $15 \mathrm{~min}$ and incubated overnight with anti-caspase 3 antibody (1:200; cat. no. 9662; Cell Signaling Technology, Inc.) at $4^{\circ} \mathrm{C}$. Detection (inclusive of secondary antibody) was performed in an automated slide staining instrument (SP9001; OriGene Technologies, Inc.) by using a 3,3'-diaminobenzidine staining kit (Maixin, Fuzhou, China) and the slices were counterstained with hematoxylin (OriGene Technologies, Inc.) at room temperature for $3 \mathrm{~min}$. All kits were used according to the manufacturer's protocol. Images 
A

$\sum_{\infty}^{\infty}$
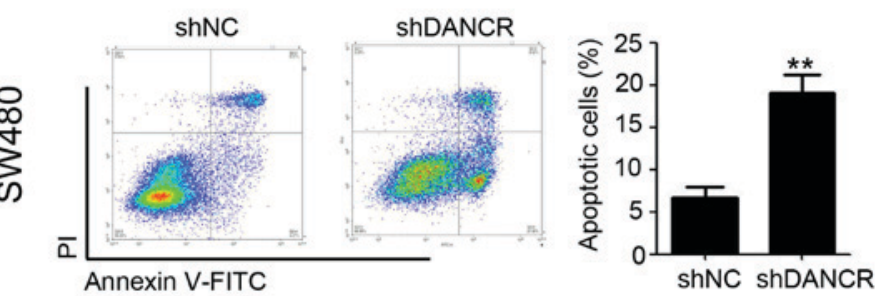

B

ShNC

ShDANCR
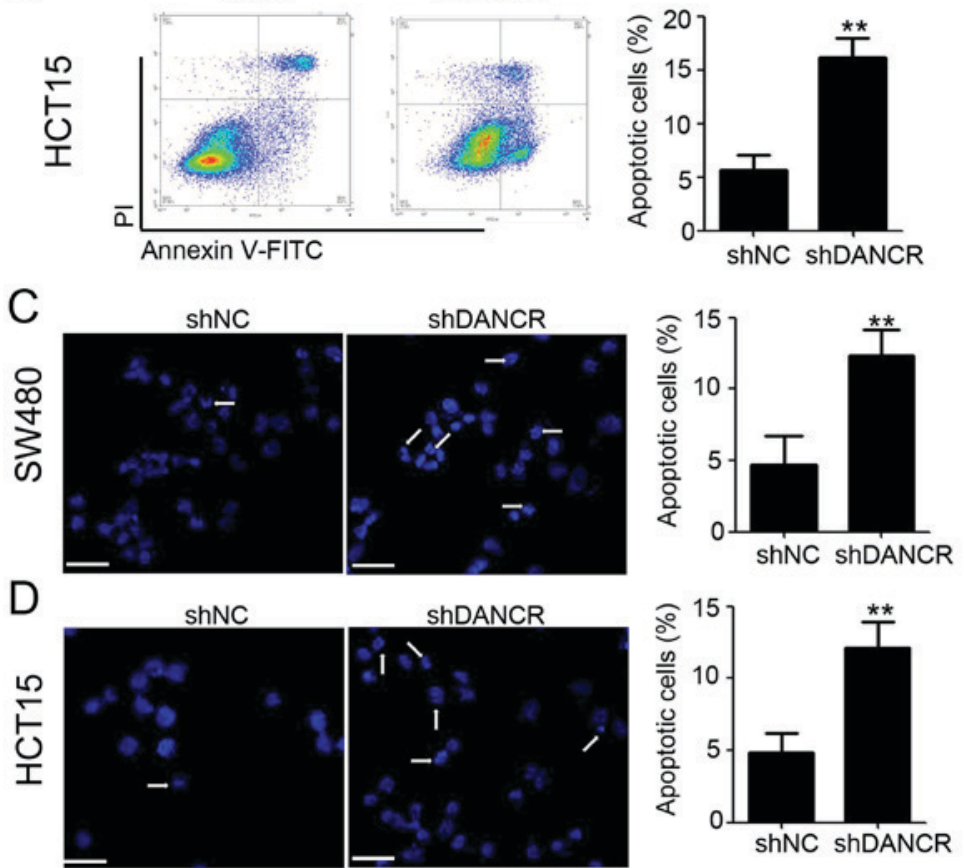

Figure 2. Silencing DANCR promotes cell apoptosis in colon cancer cells. (A) Annexin V-FITC and PI staining were performed to detect apoptotic cells in SW480-shNC and SW480-shDANCR cells, followed with flow cytometry analysis. Representative dot plots (left) and cumulative data (right) are shown (P<0.01). (B) Annexin V-FITC and PI staining were performed to detect apoptotic cells in HCT15-shNC and HCT15-shDANCR cells, followed by flow cytometry analysis. Representative dot plots (left) and cumulative data (right) are shown $(\mathrm{P}<0.01)$. (C) Hoechst 33258 staining was performed to detect apoptotic cells in SW480-shNC and SW480-shDANCR cells, followed by fluorescence capturing. Representative images (left) and cumulative data (right) are shown, scale bar $=50 \mu \mathrm{m}(\mathrm{P}<0.01)$. (D) Hoechst 33258 staining was performed to detect apoptotic cells in HCT15-shNC and HCT15-shDANCR cells, followed by fluorescence capturing. Representative images (left) and cumulative data (right) are shown, scale bar, $50 \mu \mathrm{m}(\mathrm{P}<0.01)$. ${ }^{* *} \mathrm{P}<0.01 \mathrm{vs}$. shNC. shDANCR, short hairpin differentiation antagonizing non-protein coding RNA; NC, negative control; FITC, fluorescein isothiocyanate; PI, propidium iodide; shRNA, short hairpin ribonucleic acid.

of the slices were captured with a CX51 light microscope (magnification, x20; Olympus Corporation). The caspase 3 positive, and total cell number in each frame were counted.

Statistical analysis. All data are presented as the mean \pm standard deviations. Student's t-tests were performed to compare the difference between two groups and one-way analysis of variance followed by Turkey's multiple comparisons analysis was used to compare the difference among multi-groups. SPSS software (version 21.0, IBM Corp., Armonk, NY, USA), was used. $\mathrm{P}<0.05$ was considered to indicate a statistically significant difference.

\section{Results}

Silencing DANCR inhibits colon cancer cell growth in vitro. To determine whether DANCR serves a pivotal role in colon cancer, we investigated the expression levels of lncRNA DANCR in several human colon cancer cell lines. Healthy human epithelial cells (HCoEpiC) were used as a control. It was revealed that DANCR was overexpressed in all the six colon cancer cell lines compared with HCoEpiC cells (Fig. 1A). Among the colon cancer cell lines, SW480 and HCT15 cells revealed a $>10$-fold increase in DANCR expression level (Fig. 1A). To investigate the biological effect of DANCR in colon cancer, lentivirus-based shRNA targeting DANCR was used to infect SW480 and HCT15 cells. Following puromycin selection, DANCR expression in SW480 and HCT15 cells that had been infected with shDANCR was decreased compared with shNC group (Fig. 1B and C). As a result, silencing DANCR significantly decreased SW480 and HCT15 cell proliferation (Fig. 1D and E) and colony formation (Fig. 1F and G), respectively. These data suggested that DANCR functions as an oncogene in colon cancer.

Silencing DANCR promotes cell apoptosis. To determine the potential mechanism of DANCR regulation of colon cancer cell proliferation, flow cytometry with Annexin V and PI staining was performed to detect cell apoptosis and Annexin $\mathrm{V}$ positive and PI negative cells were considered to be apoptotic cells. As shown in Fig. 2A and B, more apoptotic cells were observed in SW480-shDANCR (Fig. 2A; shDANCR vs. shNC: $19.07 \pm 1.22$ vs. 
A
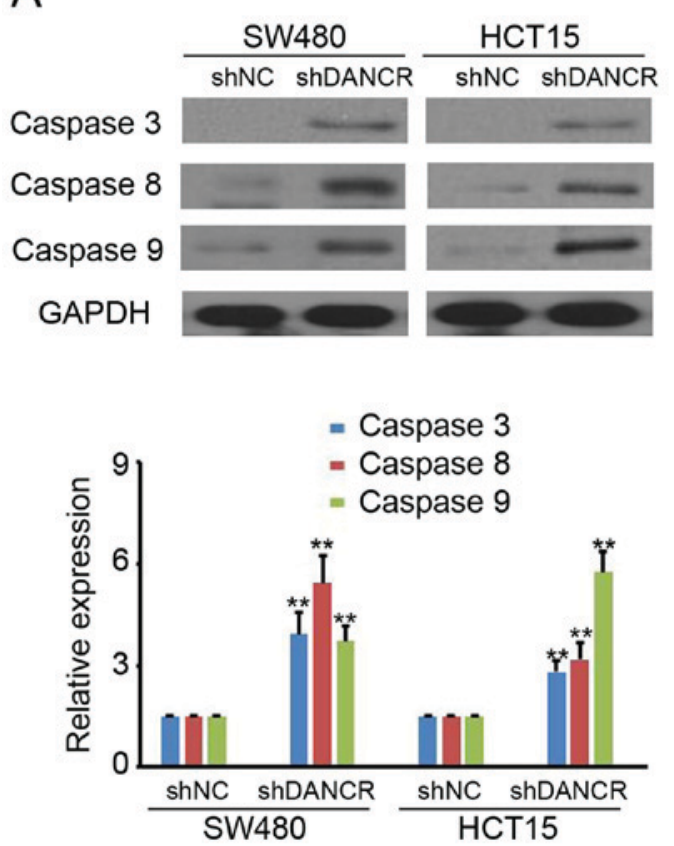

B
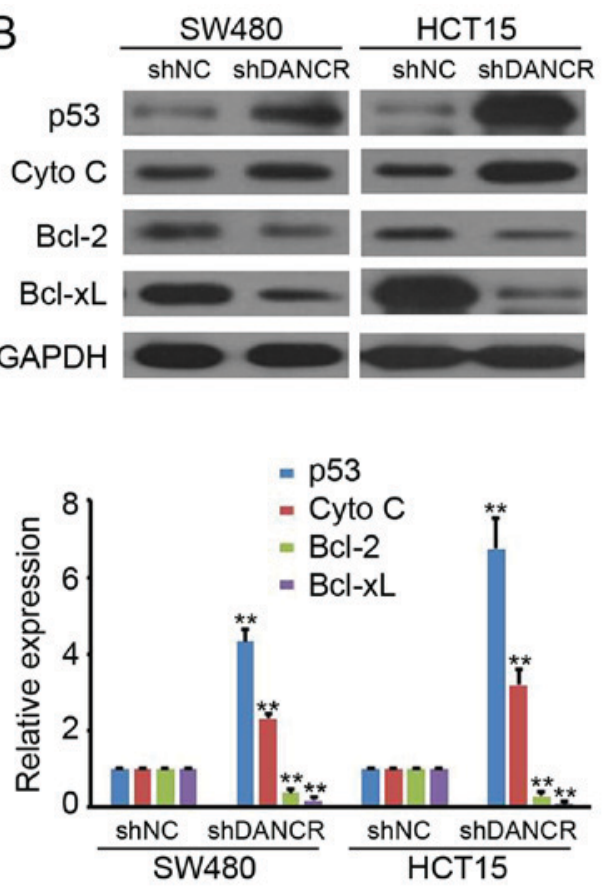

Figure 3. Silencing DANCR regulates caspase protein expression. (A) The expression of caspase 3, 8 and 9 in SW480-shNC, SW480-shDANCR HCT15-shNC and HCT15-shDANCR cells was detected by western blot analysis. GAPDH was used as a loading control. The relative expression was analyzed (P<0.01). (B) The expression of p53, Cyto C, Bcl-2 and Bcl-xL in SW480-shNC, SW480-shDANCR HCT15-shNC and HCT15-shDANCR cells were detected by western blot analysis. GAPDH was used as a loading control. The relative expression was analyzed $(\mathrm{P}<0.01){ }^{* *} \mathrm{P}<0.01 \mathrm{vs}$. shNC. shDANCR, short hairpin differentiation antagonizing non-protein coding RNA; NC, negative control; shRNA, short hairpin ribonucleic acid; Cyto C, cytochrome $c$; Bcl-2, B-cell lymphoma 2; Bcl-xL, B-cell lymphoma-extra-large.
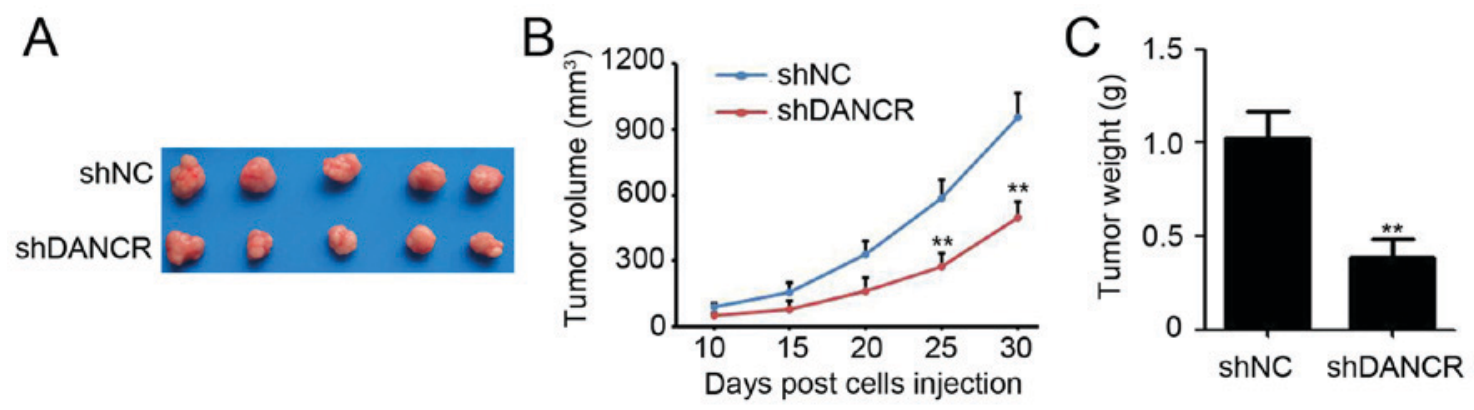

Figure 4. Silencing DANCR impairs colon tumor growth in vivo. (A) SW480-shNC and SW480-shDANCR cells were injected subcutaneously into BALB/c female mice $(\mathrm{n}=5)$. After 30 days, the mice were sacrificed and the tumors were visualized. (B) Tumor volume (ellipsoids) was measured every five days. The cumulative growth of SW480-shNC and SW480-shDANCR xenograft tumors were plotted (P<0.01). (C) The weight of SW480-shNC and SW480-shDANCR xenograft tumors were analyzed $(\mathrm{P}<0.01) .{ }^{* *} \mathrm{P}<0.01$ vs. shNC. shDANCR, short hairpin differentiation antagonizing non-protein coding RNA; NC, negative control; shRNA, short hairpin ribonucleic acid.

6.72 $\pm 0.71 \%$ ) and HCT15-shDANCR cells (Fig. 2B; shDANCR vs. shNC: $16.17 \pm 1.02$ vs. $5.63 \pm 0.82 \%)$, compared with shNC cells. Hoechst 33258 was also used to detect apoptotic cells and, in concordance with Annexin V/PI data, indicated that DANCR knockdown promotes cell apoptosis in SW480 (Fig. 2C) and HCT15 (Fig. 2D) cells. Collectively, these results suggested that silencing DANCR promotes cell apoptosis in colon cancer cells.

Silencing DANCR regulates caspase protein and upstream protein expression in colon cancer cells. To further determine the molecular mechanism behind the antitumor activity of DANCR knockdown in colon cancer cells, western blotting was employed to investigate the expression of caspase proteins and certain upstream proteins. It was revealed that silencing DANCR significantly induces caspase 3 , caspase 8 and caspase 9 expression, which demonstrated low/no basal expression levels, in SW480 and HCT15 cells (Fig. 3A). Furthermore, shDANCR was revealed to promote p53 and cyto $\mathrm{C}$ expression and inhibit Bcl-2 and Bcl-xL expression in SW480 and HCT15 cells (Fig. 3B). These results suggested that silencing DANCR regulates caspase protein and upstream protein expression in colon cancer cells.

Silencing DANCR impairs colon tumor growth in vivo. To investigate the effect of DANCR on colon cancer growth in vivo, SW480 cells that were stably infected with shDANCR and shNC were subcutaneously injected into the right flank of 6-week-old female BALB/c nude mice. After 30 days, the mice were sacrificed and the tumors were visualized (Fig. 4A). The results revealed that tumors generated from SW480 cells infected with shDANCR grew significantly slower than the negative control, evidenced by the growth curve (Fig. 4B; 
A

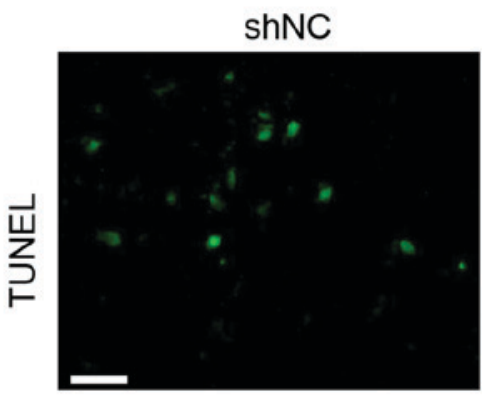

B

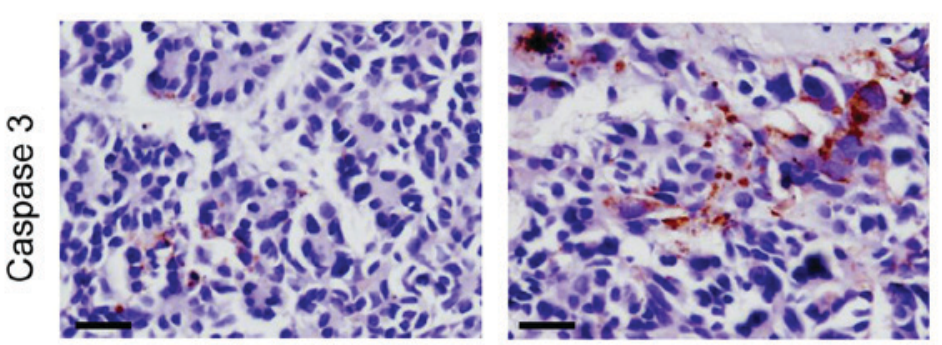

ShDANCR
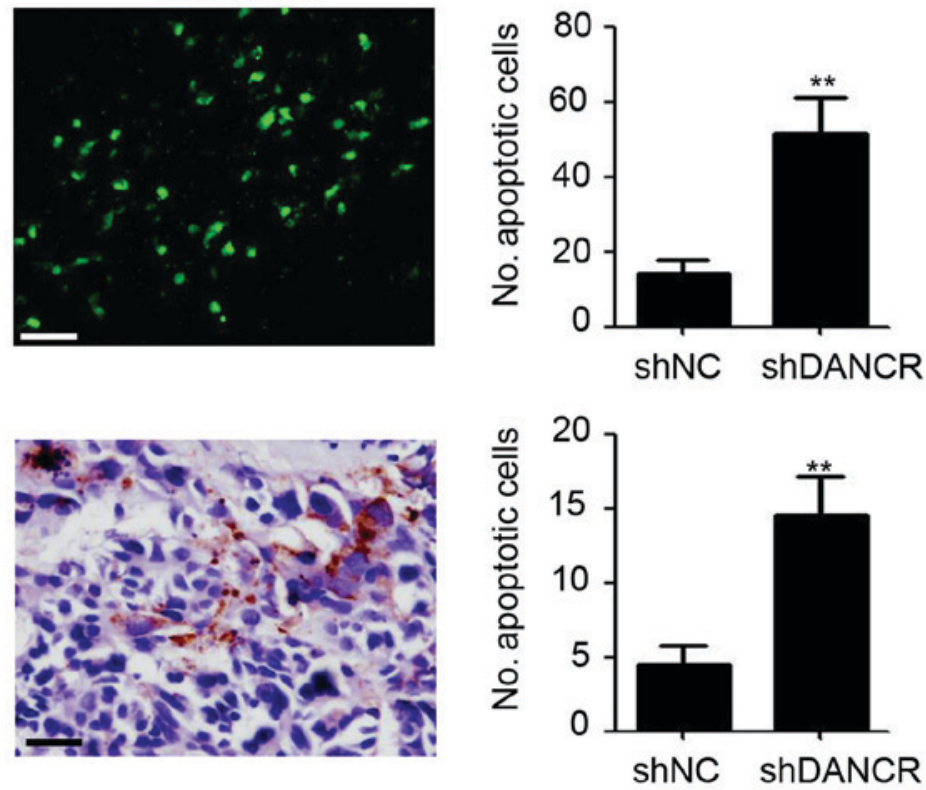

Figure 5. Silencing DANCR promotes tumor cell apoptosis and caspase 3 expression. (A) The apoptotic cells in SW480-shNC and SW480-shDANCR tumor tissues were detected by TUNEL assay. The number of apoptotic cells per frame was analyzed. Representative images (left) and cumulative data (right) are shown; scale bar, $50 \mu \mathrm{m}(\mathrm{P}<0.01)$. (B) The expression of caspase 3 in SW480-shNC and SW480-shDANCR tumor tissues was detected by immunohistochemical staining. The number of Caspase 3 positive cells in per frame was analyzed. Representative images (left) and cumulative data (right) are shown; scale bar, $50 \mu \mathrm{m}(\mathrm{P}<0.01) .{ }^{* *} \mathrm{P}<0.01$ vs. shNC. shDANCR, short hairpin differentiation antagonizing non-protein coding RNA; NC, negative control; shRNA, short hairpin ribonucleic acid; TUNEL, terminal deoxynucleotidyl transferase dUTP nick end labeling.

final tumor volume of shNC vs. shDANCR: $959.2 \pm 107.3$ vs. $\left.498.5 \pm 73.4 \mathrm{~mm}^{3}\right)$. Furthermore, silencing DANCR also caused a $58.7 \%$ reduction in the weight of SW480 cell-tumor (Fig. 4C; shNC vs. shDANCR: $1.04 \pm 0.13$ vs. $0.43 \pm 0.06 \mathrm{~g}$ ). These results suggested that DANCR was capable of regulating colon tumor growth generated from SW480 cells.

Silencing DANCR promotes tumor cell apoptosis and caspase 3 expression. As DANCR-knockdown appeared to promote cell apoptosis through the induction of caspase protein expression in colon cancer, the apoptotic cell number, and caspase 3 protein expression in SW480 tumors was further investigated. As shown in Fig. 5A, more apoptotic cells were observed in SW480-shDANCR tumors by TUNEL assay, compared with SW480-shNC tumors. Concurrently, an almost negligible number of caspase 3-positive tumor cells were observed in SW480-shNC tumor tissues (Fig. 5B). Of note, DANCR knockdown significantly promoted caspase 3 expression in SW480 tumors (Fig. 5B). These results provided further evidence for the role of DANCR knockdown in the induction of colon cancer cell apoptosis.

\section{Discussion}

Due to their critical roles in the development process, cellular homeostasis, genomic imprinting and pluripotency of embryonic stem cells (9), lncRNAs are involved in the carcinogenesis, disease progression, metastasis or chemoresistance of human cancer types $(14,15)$. In the present study, it was demonstrated that IncRNA DANCR was overexpressed in almost all colon cancer cell lines (Fig. 1). Silencing DANCR significantly inhibited cell proliferation (Fig. 1), colony formation (Fig. 1) and tumor growth (Fig. 4), and induced apoptosis (Fig. 2) through regulating caspase proteins (Figs. 3 and 5) and the expression of upstream proteins (Fig. 3). The present study provides evidence of the functional role and mechanism of DANCR in colon cancer.

In a previous study by Sha et al (15), DANCR expression was revealed to be increased in triple-negative breast cancer (TNBC) tissues compared with adjacent normal tissues in 63 TNBC specimens. Patients with higher DANCR expression correlated with worse tumor-node-metastasis stages as well as a shorter overall survival (OS) using Kaplan-Meier analysis (15). DANCR was upregulated in the tumor tissues and plasma of patients with HCC, and its expression was highly correlated with microvascular and liver capsule invasion in hepatocellular carcinoma (HCC) (18). Further receiver operating characteristic analysis demonstrated that plasma DANCR exhibited significantly increased discriminatory power for differentiating patients with HCC from HVs and non-HCC patients compared with a-fetoprotein, which has been used as a biomarker for HCC diagnosis (18). In osteosarcoma tissues, DANCR was consistently significantly increased and its expression was an independent poor prognostic factor (21). DANCR was demonstrated to be increased in colorectal cancer (CRC) tissues compared with that in adjacent normal tissues and patients with high DANCR expression had a shorter OS and disease-free survival (DFS) compared with the low DANCR expression group (19). Of note, in a multivariate Cox model, DANCR was demonstrated to be an independent poor prognostic factor for OS and DFS in CRC (19). The present study, also demonstrated an increased expression of DANCR in the majority of colon cancer cell lines that were investigated (Fig. 1). These results are consistent with the findings by Liu et al (19) in patients with colon cancer. 
DANCR has been demonstrated to have multiple functional roles in regulating cell fate. In synovium-derived MSCs, overexpression of DANCR can promote the proliferation and chondrogenesis of MSCs (22). Furthermore, DANCR functions as an oncogene in various types of cancer. In HCC, DANCR markedly increased stemness features of cancer cells to promote tumorigenesis and intra/extra-hepatic tumor colonization (23). In vivo xenograft experiments demonstrated that knockdown of DANCR in MDA-MB-231 cells reduced tumor progression significantly through inhibiting cell proliferation and invasion, and fewer cancer stem cells were observed in DANCR-knocked down MDAMB-231 cells (15). DANCR also promotes prostate cancer invasion and metastasis through repressing the expression of TIMP2/3 (17). Of note, DANCR could suppress 7860 and $\mathrm{ACHN}$ proliferation, migration, and invasion, and induce apoptosis in renal cell carcinoma (24). In the present study, we demonstrated that DANCR-knockdown significantly inhibited cell proliferation, colony formation, and tumor growth in vitro and in vivo (Figs. 1 and 4). To the best of our knowledge, the present study is the first to provide evidence for understanding how knockdown of DANCR promotes apoptosis in colon cancer cells, which contradicts previous findings in renal cell carcinoma (24). The diverse roles of DANCR in apoptosis differed between the cells investigated in the present study.

In a previous study, the role of DANCR in regulating the stemness of HCC relied largely on an association with, and regulation of, CTNNB1 (23). In TNBC, knockdown of DANCR was associated with increased binding of EZH2 to the promoters of CD44 and ABCG2 (15). MiR-1305 serves as a downstream target of DANCR during the induction of cell proliferation and chondrogenic differentiation of synovium-derived MSCs (25). Components of the Wnt/b-catenin signaling pathway were also downstream targets of DANCR during odontoblast-like differentiation of human dental pulp cells. This was demonstrated by a decrease in the expression levels of p-GSK-3b and b-catenin upon DANCR overexpression (26). The present study also demonstrated that DANCR-knockdown promoted apoptosis in colon cancer through caspases (Fig. 3). However, further investigations are needed to determine the direct target of DANCR during the regulation of apoptosis in colon cancer.

In summary, the present study highlights the potential role of DANCR in regulating colon cancer growth in vitro and in vivo. We demonstrated that caspase proteins mediated apoptosis. These findings suggested that DANCR may be a novel therapeutic target in colon cancer. However, further investigations are required to determine the direct target of DANCR in the regulation of apoptosis in colon cancer.

\section{Acknowledgements}

Not applicable.

\section{Funding}

No funding was received.

\section{Availability of data and materials}

All data generated and/or analyzed during this study are included in this published article.

\section{Authors' contributions}

XJY and JJZ were involved in the acquisition of the data. WJC, GGZ and WW were involved in the analysis and interpretation of the data. HCT was involved in the conception and design of the present study.

\section{Ethics approval and consent to participate}

The present animal study was approved by the Ethics Committee of Dongtai Municipal People's Hospital of Nantong University and complied with the animal guidelines.

\section{Consent for publication}

Not applicable.

\section{Competing interests}

The authors declare that they have no competing interests.

\section{References}

1. Siegel RL, Miller KD and Jemal A: Cancer statistics, 2017. CA Cancer J Clin 67: 7-30, 2017.

2. Siegel RL, Miller KD, Fedewa SA, Ahnen DJ, Meester RGS, Barzi A and Jemal A: Colorectal cancer statistics, 2017. CA Cancer J Clin 67: 177-193, 2017.

3. Dai L, Cui X, Zhang X, Cheng L, Liu Y, Yang Y, Fan P, Wang Q, Lin Y, Zhang J, et al: SARI inhibits angiogenesis and tumour growth of human colon cancer through directly targeting ceruloplasmin. Nat Commun 7: 11996, 2016.

4. Zhang Y, Lin C, Liao G, Liu S, Ding J, Tang F, Wang Z, Liang X, Li B, Wei Y, et al: MicroRNA-506 suppresses tumor proliferation and metastasis in colon cancer by directly targeting the oncogene EZH2. Oncotarget 6: 32586-32601, 2015.

5. Zeng M, Zhu L, Li L and Kang C: miR-378 suppresses the proliferation, migration and invasion of colon cancer cells by inhibiting SDAD1. Cell Mol Biol Lett 22: 12, 2017.

6. Frouws MA, Reimers MS, Swets M, Bastiaannet E, Prinse B, van Eijk R, Lemmens VE, van Herk-Sukel MP, van Wezel T, Kuppen PJ, et al: The Influence of BRAF and KRAS mutation status on the association between aspirin use and survival after colon cancer diagnosis. PLoS One 12: e0170775, 2017.

7. Wilusz JE: Long noncoding RNAs: Re-writing dogmas of RNA processing and stability. Biochim Biophys Acta 1859: 128-138, 2016.

8. Lam MT, Li W, Rosenfeld MG and Glass CK: Enhancer RNAs and regulated transcriptional programs. Trends Biochem Sci 39: 170-182, 2014.

9. Boon RA, Jaé N, Holdt L and Dimmeler S: Long noncoding RNAs: From clinical genetics to therapeutic targets? J Am Coll Cardiol 67: 1214-1226, 2016.

10. Zhang J, Wang P, Wan L, Xu S and Pang D: The emergence of noncoding RNAs as Heracles in autophagy. Autophagy 13: 1004-1024, 2017.

11. Dangwal S, Schimmel K, Foinquinos A, Xiao K and Thum T: Noncoding RNAs in heart failure. Handb Exp Pharmacol 243: 423-445, 2017.

12. Bär C, Chatterjee $S$ and Thum T: Long noncoding RNAs in cardiovascular pathology, diagnosis, and therapy. Circulation 134: 1484-1499, 2016

13. Adams BD, Parsons C, Walker L, Zhang WC and Slack FJ: Targeting noncoding RNAs in disease. J Clin Invest 127: 761-771, 2017.

14. Tong $\mathrm{X}, \mathrm{Gu} \mathrm{PC}, \mathrm{Xu} \mathrm{SZ}$ and Lin XJ: Long non-coding RNA-DANCR in human circulating monocytes: A potential biomarker associated with postmenopausal osteoporosis. Biosci Biotechnol Biochem 79: 732-737, 2015.

15. Sha S, Yuan D, Liu Y, Han B and Zhong N: Targeting long non-coding RNA DANCR inhibits triple negative breast cancer progression. Biol Open 6: 1310-1316, 2017. 
16. Mao Z, Li H, Du B, Cui K, Xing Y, Zhao X and Zai S: LncRNA DANCR promotes migration and invasion through suppression of lncRNA-LET in gastric cancer cells. Biosci Rep 37: pii: BSR20171070, 2017

17. Jia J, Li F, Tang XS, Xu S, Gao Y, Shi Q, Guo W, Wang X, He D and Guo P: Long noncoding RNA DANCR promotes invasion of prostate cancer through epigenetically silencing expression of TIMP2/3. Oncotarget 7: 37868-37881, 2016.

18. Dumars C, Ngyuen JM, Gaultier A, Lanel R, Corradini N, Gouin F, Heymann D and Heymann MF: Dysregulation of macrophage polarization is associated with the metastatic process in osteosarcoma. Oncotarget 7: 78343-78354, 2016.

19. Liu Y, Zhang M, Liang L, Li J and Chen YX: Over-expression of IncRNA DANCR is associated with advanced tumor progression and poor prognosis in patients with colorectal cancer. Int J Clin Exp Pathol 8: 11480-11484, 2015.

20. Livak KJ and Schmittgen TD: Analysis of relative gene expression data using real-time quantitative PCR and the 2(-Delta Delta C(T)) method. Methods 25: 402-408, 2001.

21. Jiang N, Wang X, Xie X, Liao Y, Liu N, Liu J, Miao N, Shen J and Peng T: lncRNA DANCR promotes tumor progression and cancer stemness features in osteosarcoma by upregulating AXL via miR-33a-5p inhibition. Cancer Lett 405: 46-55, 2017.

22. Zhang L, Yang C, Chen S, Wang G, Shi B, Tao X, Zhou L and Zhao J: Long noncoding RNA DANCR Is a positive regulator of proliferation and chondrogenic differentiation in human Synovium-derived stem cells. DNA Cell Biol 36: 136-142, 2017.
23. Yuan SX, Wang J, Yang F, Tao QF, Zhang J, Wang LL, Yang Y, Liu H, Wang ZG, Xu QG, et al: Long noncoding RNA DANCR increases stemness features of hepatocellular carcinoma by derepression of CTNNB1. Hepatology 63: 499-511, 2016.

24. Jin L, Fu H, Quan J, Pan X, He T, Hu J, Li Y, Li H, Yang Y, Ye J, et al: Overexpression of long non-coding RNA differentiation antagonizing non-protein coding RNA inhibits the proliferation, migration and invasion and promotes apoptosis of renal cell carcinoma. Mol Med Rep 16: 4463-4468, 2017.

25. Zhang L, Sun X, Chen S, Yang C, Shi B, Zhou L and Zhao J: Long noncoding RNA DANCR regulates miR-1305-Smad 4 axis to promote chondrogenic differentiation of human synovium-derived mesenchymal stem cells. Biosci Rep 37: pii: BSR20170347, 2017.

26. Chen L, Song Z, Huang S, Wang R, Qin W, Guo J and Lin Z: lncRNA DANCR suppresses odontoblast-like differentiation of human dental pulp cells by inhibiting wnt/ $\beta$-catenin pathway. Cell Tissue Res 364: 309-318, 2016.

(7)(9) This work is licensed under a Creative Commons Attribution-NonCommercial-NoDerivatives 4.0 International (CC BY-NC-ND 4.0) License. 